\title{
COVID-19 Pneumonia Diagnosis Using a Simple 2D Deep Learning Framework With a Single Chest CT Image: Model Development and Validation
}

Hoon $\mathrm{Ko}^{1^{*}}$, MSc; Heewon Chung ${ }^{1 *}$, MSc; Wu Seong Kang ${ }^{2 *}$, MD, PhD; Kyung Won Kim ${ }^{3}$, MD; Youngbin Shin ${ }^{3}$, MSc; Seung Ji Kang ${ }^{4}$, MD; Jae Hoon Lee ${ }^{5}$, MD; Young Jun Kim5 ${ }^{5}$ MD; Nan Yeol Kim², MD, PhD; Hyunseok Jung, MD; Jinseok Lee ${ }^{1}, \mathrm{PhD}$

\footnotetext{
${ }^{1}$ Department of Biomedical Engineering, Wonkwang University College of Medicine, Iksan-si, Republic of Korea

${ }^{2}$ Department of Trauma Surgery, Wonkwang University Hospital, Iksan-si, Republic of Korea

${ }^{3}$ Department of Radiology and Research Institute of Radiology, Asan Image Metrics, Clinical Trial Center, Asan Medical Center, University of Ulsan College of Medicine, Seoul, Republic of Korea

${ }^{4}$ Department of Internal Medicine, Chonnam National University Medical School, Gwangju-si, Republic of Korea

${ }^{5}$ Department of Internal Medicine, Wonkwang University Hospital, Iksan-si, Republic of Korea

${ }^{6}$ Department of Radiology, Wonkwang University Hospital, Iksan-si, Republic of Korea

*these authors contributed equally
}

\section{Corresponding Author:}

Jinseok Lee, $\mathrm{PhD}$

Department of Biomedical Engineering

Wonkwang University College of Medicine

460 Iksan-daero

Iksan-si, 54538

Republic of Korea

Phone: 82638505114

Email: gonasago@wku.ac.kr

\section{Abstract}

Background: Coronavirus disease (COVID-19) has spread explosively worldwide since the beginning of 2020. According to a multinational consensus statement from the Fleischner Society, computed tomography (CT) is a relevant screening tool due to its higher sensitivity for detecting early pneumonic changes. However, physicians are extremely occupied fighting COVID-19 in this era of worldwide crisis. Thus, it is crucial to accelerate the development of an artificial intelligence (AI) diagnostic tool to support physicians.

Objective: We aimed to rapidly develop an AI technique to diagnose COVID-19 pneumonia in CT images and differentiate it from non-COVID-19 pneumonia and nonpneumonia diseases.

Methods: A simple 2D deep learning framework, named the fast-track COVID-19 classification network (FCONet), was developed to diagnose COVID-19 pneumonia based on a single chest CT image. FCONet was developed by transfer learning using one of four state-of-the-art pretrained deep learning models (VGG16, ResNet-50, Inception-v3, or Xception) as a backbone. For training and testing of FCONet, we collected 3993 chest CT images of patients with COVID-19 pneumonia, other pneumonia, and nonpneumonia diseases from Wonkwang University Hospital, Chonnam National University Hospital, and the Italian Society of Medical and Interventional Radiology public database. These CT images were split into a training set and a testing set at a ratio of 8:2. For the testing data set, the diagnostic performance of the four pretrained FCONet models to diagnose COVID-19 pneumonia was compared. In addition, we tested the FCONet models on an external testing data set extracted from embedded low-quality chest CT images of COVID-19 pneumonia in recently published papers.

Results: Among the four pretrained models of FCONet, ResNet-50 showed excellent diagnostic performance (sensitivity $99.58 \%$, specificity $100.00 \%$, and accuracy $99.87 \%$ ) and outperformed the other three pretrained models in the testing data set. In the additional external testing data set using low-quality CT images, the detection accuracy of the ResNet-50 model was the highest (96.97\%), followed by Xception, Inception-v3, and VGG16 (90.71\%, 89.38\%, and 87.12\%, respectively). 
Conclusions: FCONet, a simple 2D deep learning framework based on a single chest CT image, provides excellent diagnostic performance in detecting COVID-19 pneumonia. Based on our testing data set, the FCONet model based on ResNet-50 appears to be the best model, as it outperformed other FCONet models based on VGG16, Xception, and Inception-v3.

(J Med Internet Res 2020;22(6):e19569) doi: 10.2196/19569

\section{KEYWORDS}

COVID-19; deep learning; convolutional neural networks, transfer learning; chest CT; CT; neural network; pneumonia; artificial intelligence; diagnosis; pneumonia; scan

\section{Introduction}

The coronavirus disease (COVID-19) pandemic is currently a global health crisis; more than $1,700,000$ cases had been confirmed worldwide and more than 100,000 deaths had occurred at the time of writing this paper [1]. COVID-19, an infection caused by severe acute respiratory syndrome coronavirus 2 (SARS-CoV-2), is highly contagious and has spread rapidly worldwide. In severe cases, COVID-19 can lead to acute respiratory distress, multiple organ failure, and eventually death. Countries are racing to slow the spread of the virus by testing and treating patients in the early stage as well as quarantining people who are at high risk of exposure due to close contact with patients with confirmed infection. In addition, early diagnosis and aggressive treatment are crucial to saving the lives of patients with confirmed infection [2].

COVID-19 is typically confirmed by viral nucleic acid detection using reverse transcription-polymerase chain reaction (RT-PCR) [3]. However, the sensitivity of RT-PCR may not be sufficiently high; it ranges from $37 \%$ to $71 \%$ according to early reports [4-6]. Thus, RT-PCR can afford a substantial number of false negative results due to inadequate specimen collection, improper extraction of nucleic acid from the specimen, or collection at a too-early stage of infection. A chest computed tomography (CT) scan can be used as an important tool to diagnose COVID-19 in cases with false negative results by RT-PCR [6-9].

Recently, a multinational consensus statement from the Fleischner Society was issued to guide chest imaging during the COVID-19 pandemic in different clinical settings [6]. According to this consensus statement, in a setting such as South Korea, where detecting patients at an early stage and isolating all patients and people with high risk of exposure is essential, $\mathrm{CT}$ is a relevant screening tool due to its greater sensitivity for detecting early pneumonic changes. CT can also contribute to the management and triage of the disease by detecting severe cases. In addition, chest $\mathrm{CT}$ is noninvasive and is easy to perform in an equipped facility.

However, radiologic diagnostic support is not maintained 24 hours per day in many institutions [10]. In addition, CT may show similar imaging features between COVID-19 and other types of pneumonia, thus hampering correct diagnosis by radiologists. Indeed, in a study that evaluated radiologists' performance in differentiating COVID-19 from other viral pneumonia, the median values and ranges of sensitivity and specificity were $83 \%(67 \%-97 \%)$ and $96.5 \%(7 \%-100 \%)$, respectively [11].

The use of artificial intelligence (AI) may help overcome these issues, as AI can help maintain diagnostic radiology support in real time and with increased sensitivity $[8,12]$. In this era of worldwide crisis, it is crucial to accelerate the development of AI techniques to detect COVID-19 and to differentiate it from non-COVID-19 pneumonia and nonpneumonia diseases in CT images. Therefore, we aimed to rapidly develop an AI technique using all available CT images from our institution as well as publicly available data.

\section{Methods}

\section{Data Sets and Imaging Protocol}

This study was approved by the institutional review boards of Wonkwang University Hospital (WKUH) and Chonnam National University Hospital (CNUH). Informed consent was waived. Table 1 summarizes the training, testing, and additional validation data sets. In this study, we initially collected data from 3993 chest CT images, which were categorized into COVID-19, other pneumonia, and nonpneumonia disease groups.

For the COVID-19 data group, we used a total of 1194 chest CT images: 673 chest CT images (56.3\%, from 13 patients) from CNUH, 421 images (35.3\%, from 7 patients) from WKUH, and 100 images $(83.8 \%, 60$ patients) from the Italian Society of Medical and Interventional Radiology (SIRM) public database [13]. The 20 patients from CNUH and WKUH included 9 male patients and 11 female patients, with an average age of 59.6 years (SD 17.2). Regarding the COVID-19 data from WKUH and CNUH, all the patients with COVID-19 tested positive for the virus by RT-PCR viral detection, and the CT images were acquired between December 31, 2019 and March 25, 2020. The median period from symptom onset to the first chest CT examination was 8 days (range 2-20 days). The most common symptoms were fever (75\%) and myalgia (30\%). In addition, according to previous studies related to COVID-19 by Zhao's group [14] from January 19 and March 25, 2020, 264 low-quality chest CT images were used as additional testing data. In summary, 1194 COVID-19 images (80 patients) from WKUH, CNUH, and SRIM were split into the training data set (955 images, 80.0\%) and testing data set (239 images, 20.0\%). For the additional testing, 264 COVID-19 images (264 patients) from the low-quality image data set were used. 
Table 1. Summary of the training, testing, and additional testing data sets $(\mathrm{N}=4257)$.

\begin{tabular}{|c|c|c|}
\hline Data type, data source, and data group & Training images, $\mathrm{n}(\%)$ & Testing images, $\mathrm{n}(\%)$ \\
\hline \multicolumn{3}{|l|}{ Training and testing data } \\
\hline \multicolumn{3}{|l|}{ WKUH $^{\mathbf{a}}$} \\
\hline COVID-19 pneumonia $(n=421)$ & $337(80.0)$ & $84(20.0)$ \\
\hline Other pneumonia $(\mathrm{n}=1357)$ & $1086(80.0)$ & $271(20.0)$ \\
\hline Nonpneumonia and normal lung ( $\mathrm{n}=998)$ & $798(80.0)$ & $200(20.0)$ \\
\hline Lung cancer $(n=444)$ & $355(80.0)$ & $89(20.0)$ \\
\hline \multicolumn{3}{|l|}{$\mathrm{CNUH}^{\mathrm{b}}$} \\
\hline COVID-19 pneumonia $(n=673)$ & $538(80.0)$ & $135(20.0)$ \\
\hline \multicolumn{3}{|l|}{$\operatorname{SIRM}^{\mathrm{c}}$} \\
\hline COVID-19 pneumonia $(n=100)$ & $80(80.0)$ & $20(20.0)$ \\
\hline \multicolumn{3}{|l|}{ Additional external testing data } \\
\hline \multicolumn{3}{|l|}{ Low-quality CT images from papers } \\
\hline COVID-19 ${ }^{\mathrm{d}}$ pneumonia $(\mathrm{n}=264)$ & $0(0.0)$ & $264(100.0)$ \\
\hline
\end{tabular}

${ }^{\mathrm{a}}$ WKUH: Wonkwang University Hospital.

${ }^{\mathrm{b}} \mathrm{CNUH}$ : Chonnam National University Hospital.

${ }^{\mathrm{C}}$ SIRM: Italian Society of Medical and Interventional Radiology.

${ }^{\mathrm{d}}$ COVID-19: coronavirus disease.

For the other pneumonia data group, we selected 1357 chest CT images from 100 patients diagnosed with non-COVID-19 pneumonia at WKUH between September 1, 2019, and March 30,2020 . The average age of this group was 62.5 years (SD 17.2), with 68 male and 32 female patients. For the nonpneumonia data group, we also selected 1442 chest CT images from 126 patients who had no lung parenchymal disease or lung cancers at WKUH between January 2009 and December 2014. The average age of these patients was 47 years (SD 17), with 63 male patients (721/1442 images, 50.0\%) and 63 female patients $(721 / 1442$ images, $50.0 \%)$. The patient demographic statistics of the COVID-19 and other pneumonia groups are summarized in Table 2. In this table, other pneumonia (not COVID-19) was categorized into two different types based on clinical characteristics: 68 cases of community-acquired pneumonia (onset 48 hours before hospital admission) and 32 cases of hospital-acquired pneumonia (onset 48-72 hours after hospital admission). Of these other pneumonia patients, 24/100 $(24.0 \%)$ received laboratory confirmation of the etiology, 21 $(21.0 \%)$ were confirmed to be bacterial culture positive, 3

(3.0\%) were viral influenza positive by RT-PCR, and 76 (76.0\%) were negative. Regarding the imaging protocols, each volumetric examination contained approximately 51 to 1094 CT images, with varying slice thicknesses from 0.5 millimeters to $3 \mathrm{~mm}$. The reconstruction matrix was $512 \times 512$ pixels, with in-plane pixel spatial resolution from $0.29 \times 0.29$ to $0.98 \times 0.98$ square millimeters.

The data from WKUH, CNUH, and SIRM were randomly split with a ratio of $8: 2$ into a training set and a testing set, respectively, in a stratified fashion. In addition, the data for each group (WKUH, CNUH, and SIRM) were spread over different splits with a ratio of 8:2. The training data set was then further separated into sets used for training the model ( $80 \%$ of the training set) and for internal validation (20\% of the training set). The testing set was used only for independent testing of the developed models and was never used for training the model or for internal validation. Furthermore, we tested the trained model with the additional external validation data set of low-quality images to evaluate the external generalizability of the model. 
Table 2. Demographic data of patients with COVID-19 and other pneumonia.

\begin{tabular}{|c|c|c|c|}
\hline Characteristic & COVID-19a pneumonia $(\mathrm{n}=20)$ & Other pneumonia $(\mathrm{n}=100)$ & $P$ value \\
\hline Age (years), mean (SD) & $59.6(17.2)$ & $60.1(17.1)$ & .91 \\
\hline Male sex, n (\%) & $9(45.0)$ & $68(68.0)$ & .12 \\
\hline Community-acquired pneumonia, $\mathrm{n}(\%)$ & $20(100.0)$ & $68(68.0)$ & .007 \\
\hline Hospital-acquired pneumonia, n (\%) & $0(0.0)$ & $32(32.0)$ & \\
\hline \multicolumn{4}{|l|}{ Microbiological study, n (\%) } \\
\hline COVID-19 positive (RT-PCR ${ }^{\mathrm{b}}$ ) & $20(100.0)$ & $0(0.0)$ & $<.001$ \\
\hline Other virus positive (influenza) & $0(0.0)$ & $3(3.0)$ & \\
\hline Bacterial culture positive & $0(0.0)$ & $21(21.0)$ & \\
\hline Unknown & $0(0.0)$ & $76(76.0)$ & \\
\hline \multicolumn{4}{|l|}{ Human radiologist's diagnosis, $n(\%)$} \\
\hline $\begin{array}{l}\text { Atypical pneumonia or } \\
\text { viral pneumonia }\end{array}$ & $20(100.0)$ & $15(15.0)$ & $\mathrm{N} / \mathrm{A}^{\mathrm{c}}$ \\
\hline Pneumonia & $0(0.0)$ & $77(77.0)$ & \\
\hline Aspiration pneumonia & $0(0.0)$ & $26(26.0)$ & \\
\hline Necrotizing pneumonia & $0(0.0)$ & $5(5.0)$ & \\
\hline Tuberculosis & $0(0.0)$ & $5(5.0)$ & \\
\hline Empyema & $0(0.0)$ & $3(3.0)$ & \\
\hline Emphysema & $0(0.0)$ & $9(9.0)$ & \\
\hline Bronchiectasis & $0(0.0)$ & $4(4.0)$ & \\
\hline Interstitial lung disease & $0(0.0)$ & $1(1.0)$ & \\
\hline
\end{tabular}

${ }^{\mathrm{a} C O V I D-19:}$ coronavirus disease.

${ }^{\mathrm{b}}$ RT-PCR: reverse transcription-polymerase chain reaction.

${ }^{\mathrm{c}} \mathrm{N} / \mathrm{A}$ : not applicable.

\section{Preprocessing}

For the data acquired from WKUH and CNUH, we converted Digital Imaging and Communications in Medicine (DICOM) images to one-channel grayscale PNG images to standardize the image file format, as the images in the low-quality image data set were in PNG format. To minimize the information loss, we first displayed the DICOM images using a lung window with a 1500 Hounsfield unit window width and a $-600 \mathrm{HU}$ window level $[15,16]$ and converted the images to PNG format. Subsequently, we rescaled the images to a size of $256 \times 256$ pixels and normalized the pixel values to a range between 0 and 1. All of the converted PNG format images were confirmed by three radiologists to determine any loss of image information related to pulmonary diseases. For the data from SIRM, the original JPEG format was also reformatted to the PNG format,

and the images were rescaled and normalized in the same manner. For the low-quality image data set, we also rescaled and normalized the images. In this study, no further preprocessing such as lung segmentation was performed.

\section{Image Augmentation}

To reduce overfitting of the training image data, we employed two distinct forms of data augmentation: image rotation and zoom. In the data augmentation method for the rotation, angles of rotation between $-10^{\circ}$ and $10^{\circ}$ were randomly selected. Regarding the zoom, the range was randomly selected between 90\% (zoom-in) and $110 \%$ (zoom-out). Either rotation or zoom was randomly selected 10 times for each training image. By applying data augmentation, we increased the number of images in the training data set to 31,940 . Table 3 shows the number of augmented images for training in each group. 
Table 3. Augmented images for training in each group $(\mathrm{N}=31,940)$.

\begin{tabular}{ll}
\hline Data source and group & Augmented images for training, $\mathrm{n}(\%)$ \\
\hline WKUHx $^{\mathbf{a}}$ & $3370(10.6)$ \\
COVID-19 $^{\text {b }}$ pneumonia & $10,860(34.0)$ \\
Other pneumonia & $7890(24.7)$ \\
$\quad$ Nonpneumonia and normal lung & $3550(11.1)$ \\
Lung cancer & \\
CNUH $^{\mathbf{c}}$ & $5380(16.8)$ \\
COVID-19 pneumonia $^{\text {SIRM }}$ & \\
COVID-19 pneumonia $^{\mathbf{d}}$ & $800(2.5)$ \\
\hline
\end{tabular}

${ }^{\mathrm{a}}$ WKUH: Wonkwang University Hospital.

${ }^{\mathrm{b}}$ COVID-19: coronavirus disease

${ }^{\mathrm{c}} \mathrm{CNUH}$ : Chonnam National University Hospital.

${ }^{\mathrm{d}}$ SIRM: Italian Society of Medical and Interventional Radiology.

\section{The Fast-Track COVID-19 Classification Network for COVID-19 Classification}

We developed a simple 2D deep learning framework based on a single chest CT image for the classification of COVID-19 pneumonia, other pneumonia, and nonpneumonia, named the fast-track COVID-19 classification network (FCONet; Figure 1). FCONet was developed by transfer learning based on one of the following four pretrained convolutional neural network (CNN) models as a backbone: VGG16 [17], ResNet-50 [18],
Inception-v3 [19], and Xception [20]. Transfer learning is a popular method in computer vision because it enables an accurate model to be built in a short time [21]. With transfer learning, instead of starting the learning process from an optimal model search, one can start it from patterns that were learned when solving a different problem. To minimize the training time, we initially used the predefined weights for each CNN architecture, which were further updated through the learning process of classification of COVID-19 pneumonia, other pneumonia, and nonpneumonia.

Figure 1. Scheme of FCONet, a 2D deep learning framework based on a single chest CT image for the classification of COVID-19 pneumonia, other pneumonia, and non-pneumonia. COVID-19: coronavirus disease.

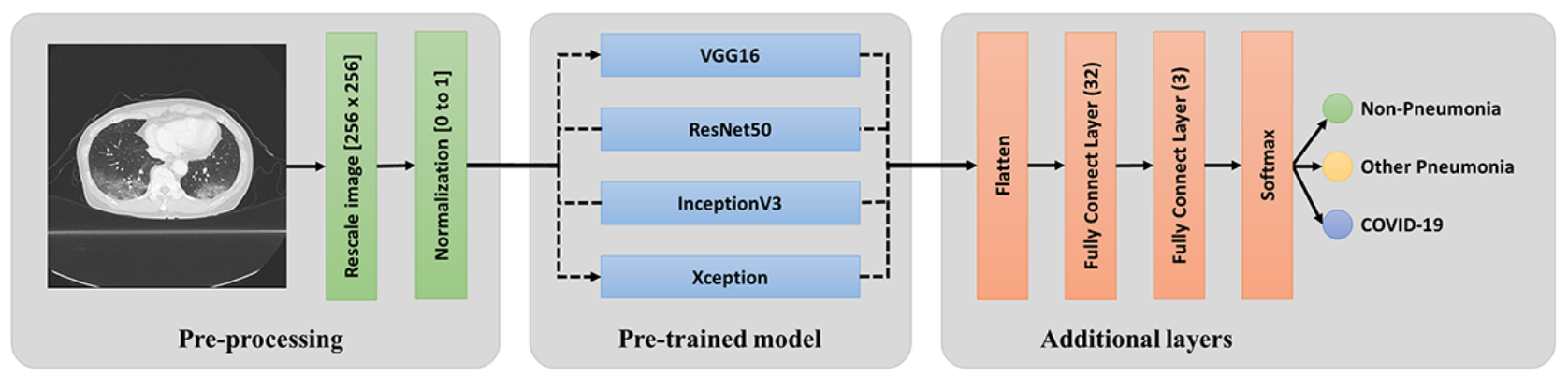

\section{Input Layer}

After the simple preprocessing stage, in the input layer, we arranged three channels $(256 \times 256 \times 3$ pixels $)$ by copying the one-channel normalized image. The three-channel images were fed into the pretrained model layers.

\section{Pretrained Model Layers}

A pretrained model is a model that was trained on a large benchmark data set to solve a similar problem to the one we want to solve. In the pretrained model layers, we included one of the four pretrained models (VGG16, ResNet-50, Inception-v3, and Xception). Each model comprises two parts: a convolutional base and a classifier. The convolutional base is composed of a stack of convolutional and pooling layers to generate features from the images. The role of the classifier is to categorize the image based on the extracted features. In our pretrained model layers, we retained the convolutional base and removed the classifier, which was replaced by another classifier for COVID-19, other pneumonia, or nonpneumonia.

\section{Additional Layers}

The activations from the pretrained model layers were fed into the additional layers. The layers acted as classifiers for COVID-19 pneumonia, other pneumonia, and nonpneumonia. In the additional layers, we first flattened the activations and connected two fully connected layers; one of the layers consisted of 32 nodes, and the other consisted of three nodes. Subsequently, the three activations from the second fully connected layer were fed into a SoftMax layer, which provided 
the probability for each of COVID-19, other pneumonia, and nonpneumonia.

\section{Implementation}

We implemented FCONet using the TensorFlow package, which provides a Python application programming interface (API) for tensor manipulation. We also used Keras as the official front end of TensorFlow. We trained the models with the Adam optimizer [22] and the categorical cross-entropy cost function with a learning rate of 0.0001 and a batch size of 32 on a GeForce GTX 1080 Ti graphics processing unit (NVIDIA). For the performance evaluation, 5-fold cross-validation was performed to confirm the generalization ability. The training data set $(\mathrm{N}=31,940)$ was randomly shuffled and divided into five equal groups in a stratified manner. Subsequently, four groups were selected to train the model, and the remaining group was used for validation. This process was repeated five times by shifting the internal validation group. Next, we averaged the mean validation costs of the five internal validation groups according to each epoch and found the optimal epoch that provides the lowest validation cost. Then, we retrained the model using the entire training data set with the optimal epoch. The testing data set was evaluated only after the model was completely trained using the training data set. This holdout method provides an unbiased evaluation of the final model by avoiding overfitting to the training data set.

\section{Performance Evaluation and Statistical Evaluation}

For each of the different four pretrained models (VGG16, ResNet-50, Inception-v3, and Xception) in FCONet, we evaluated the classification performance based on sensitivity, specificity, and accuracy. More specifically, we calculated true positives (TP), false positives (FP), true negatives (TN), and false negatives (FN) based on the groups of COVID-19 pneumonia, other pneumonia, and nonpneumonia. For each group, we expressed measure metrics with the subscripts covid for COVID-19, other for other pneumonia, and none for nonpneumonia, as

$$
\begin{gathered}
\text { Sensitivity }_{\text {covid }}=\frac{T P_{\text {covid }}}{T P_{\text {covid }}+F N_{\text {covid }}} \\
\text { Specificity }_{\text {covid }}=\frac{T N_{\text {covid }}}{T N_{\text {covid }}+F P_{\text {covid }}} \\
\text { Accuracy }_{\text {covid }}=\frac{T P_{\text {covid }}+T N_{\text {covid }}}{\text { Total number of testing data }}
\end{gathered}
$$

where $T P_{\text {covid }}$ is the number of COVID-19 testing data correctly classified as COVID-19, $T N_{\text {covid }}$ is the number of non-COVID-19 testing data correctly classified as non-COVID-19, FP $P_{\text {covid }}$ is the number of non-COVID-19 testing data misclassified as COVID-19, and $F N_{\text {covid }}$ is the number of COVID testing data misclassified as non-COVID-19.
Here, non-COVID-19 testing data include other pneumonia and nonpneumonia. Note that the same calculations were applied to the other pneumonia and nonpneumonia testing data as

$$
\begin{aligned}
& \text { Sensitivity }_{\text {other }}=\frac{T P_{\text {other }}}{T P_{\text {other }}+F N_{\text {other }}} \\
& \text { Specificity }_{\text {other }}=\frac{T N_{\text {other }}}{T N_{\text {other }}+F P_{\text {other }}} \\
& \text { Accuracy }_{\text {other }}=\frac{T P_{\text {other }}+T N_{\text {other }}}{\text { Total number of testing data }} \\
& \text { Sensitivity }_{\text {none }}=\frac{T P_{\text {none }}}{T P_{\text {none }}+F N_{\text {none }}} \\
& \text { Specificity }_{\text {none }}=\frac{T N_{\text {none }}}{T N_{\text {none }}+F P_{\text {none }}} \\
& \text { Accuracy }_{\text {none }}=\frac{T P_{\text {none }}+T N_{\text {none }}}{\text { Total number of testing data }}
\end{aligned}
$$

We also plotted the receiver operating characteristic (ROC) curve and calculated the area under the curve (AUC) for each of the four different models. Additionally, statistical analysis was performed using MATLAB (R2013b). Analysis of variance (ANOVA) was used to compare differences among COVID-19 pneumonia, non-COVID-19 pneumonia, and nonpneumonia groups. A $P$ value less than .001 was considered to indicate statistical significance.

\section{Results}

The performance of the FCONet models based on the four pretrained models in the classification of COVID-19 pneumonia, other pneumonia, and nonpneumonia is summarized in Table 4. We compared the metric values of sensitivity (\%), specificity $(\%)$, and accuracy (\%) as well as the AUCs of the four FCONet models based on VGG16, ResNet-50, Inception-v3, and Xception. Based on the testing data, the FCONet models based on ResNet-50, VGG16, and Xception showed excellent classification performance; all these models provided AUC values ranging from 0.99 to 1.00 . More specifically, with ResNet-50, the sensitivity, specificity, and accuracy for classifying COVID-19 pneumonia were $99.58 \%, 100 \%$, and $99.87 \%$, respectively. With VGG16, the sensitivity, specificity, and accuracy for classifying COVID-19 pneumonia were $100 \%$, $99.64 \%$, and $99.75 \%$, respectively. With Xception, the sensitivity, specificity, and accuracy for COVID-19 pneumonia classification were $97.91 \%, 99.29 \%$, and $98.87 \%$, respectively. For other pneumonia and nonpneumonia, the sensitivity, specificity, and accuracy ranged from $97 \%$ to $100 \%$ when ResNet-50, VGG16, or Xception was used as the backbone in FCONet. On the other hand, Inception-v3-based FCONet provided relatively low sensitivity, specificity, and accuracy values for all groups of COVID-19 pneumonia, other pneumonia, and nonpneumonia $(P<.001)$. 
Table 4. Performance of the FCONet frameworks based on the four pretrained models on the testing data set.

\begin{tabular}{|c|c|c|c|c|c|}
\hline Model and data group & Sensitivity, \% & Specificity, $\%$ & Accuracy, $\%$ & $\mathrm{AUC}^{\mathrm{a}}$ & $P$ value \\
\hline ResNet-50 & & & & & $<.001$ \\
\hline COVID $-19^{\mathrm{b}}$ pneumonia & 99.58 & 100.00 & 99.87 & 1.00 & \\
\hline Other pneumonia & 97.42 & 99.81 & 99.00 & 0.99 & \\
\hline Nonpneumonia & 100.00 & 98.63 & 99.12 & 0.99 & \\
\hline VGG16 & & & & & $<.001$ \\
\hline COVID-19 pneumonia & 100.00 & 99.64 & 99.75 & 1.00 & \\
\hline Other pneumonia & 100.00 & 99.81 & 99.87 & 0.99 & \\
\hline Nonpneumonia & 100.00 & 99.80 & 99.87 & 0.99 & \\
\hline Xception & & & & & $<.001$ \\
\hline COVID-19 pneumonia & 97.91 & 99.29 & 98.87 & 0.99 & \\
\hline Other pneumonia & 98.52 & 99.05 & 98.87 & 0.99 & \\
\hline Nonpneumonia & 100.00 & 100.00 & 100.00 & 1.00 & \\
\hline Inception-v3 & & & & & $<.001$ \\
\hline COVID-19 pneumonia & 88.28 & 97.68 & 94.87 & 0.97 & \\
\hline Other pneumonia & 94.10 & 95.83 & 95.24 & 0.98 & \\
\hline Nonpneumonia & 98.27 & 97.25 & 97.62 & 0.99 & \\
\hline
\end{tabular}

a AUC: area under the curve.

${ }^{\mathrm{b}}$ COVID-19: coronavirus disease.

The confusion matrices and ROC curves for the pretrained models on the testing data set are presented in Figures 2-5. More specifically, ResNet-50 exhibited $T P_{\text {covid }}, T P_{\text {other }}$, and $T P_{\text {none }}$ of 238/239, 268/271, and 289/289, respectively (Figure 2). VGG16 exhibited $T P_{\text {covid }}, T P_{\text {other }}$, and $T P_{\text {none }}$ of $234 / 239,269 / 271$, and 289/289, respectively (Figure 3). Xception exhibited $T P_{\text {covid }}$, $T P_{\text {other }}$, and $T P_{\text {none }}$ of $188 / 239,257 / 271$, and 289/289, respectively (Figure 4). Inception-v3 exhibited $T P_{\text {covid }}, T P_{\text {other }}$, and $T P_{\text {none }}$ of $211 / 239,255 / 271$, and 284/289, respectively
(Figure 5). For the three models of ResNet-50, VGG16, and Xception, the values of AUC were very close to 1 because the predicted probability values were provided as values close to 1 for correct labeling and values close to 0 for incorrect labeling.

On the additional external validation data set, which comprised low-quality CT images of COVID-19 pneumonia embedded in recently published papers, the detection accuracy of ResNet-50 was the highest with $96.97 \%$, followed by Xception $(90.71 \%$ ), Inception-v3 (89.38\%), and VGG16 (87.12\%) (Table 5).

Figure 2. Confusion matrix and ROC curve in FCONet using ResNet-50; COVID-19: coronavirus disease; ROC: receiver operating characteristic.

\section{Confusion Matrix (ResNet50)}

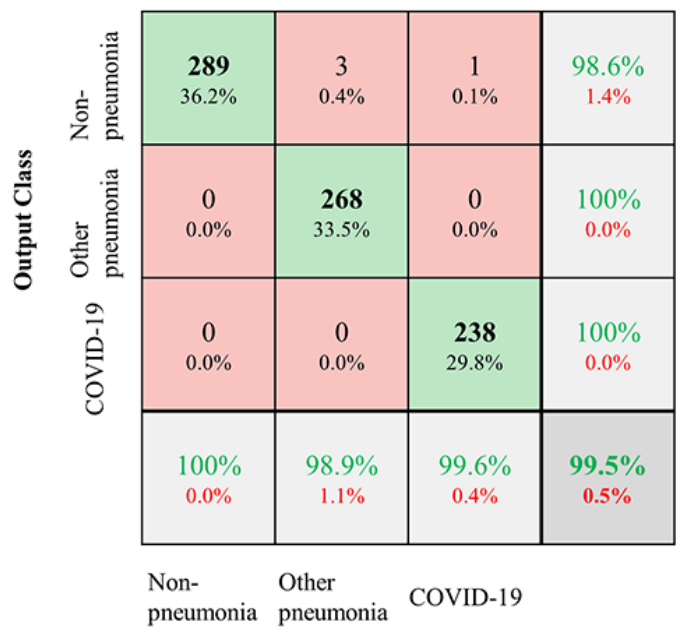

Target Class
ROC (ResNet50)

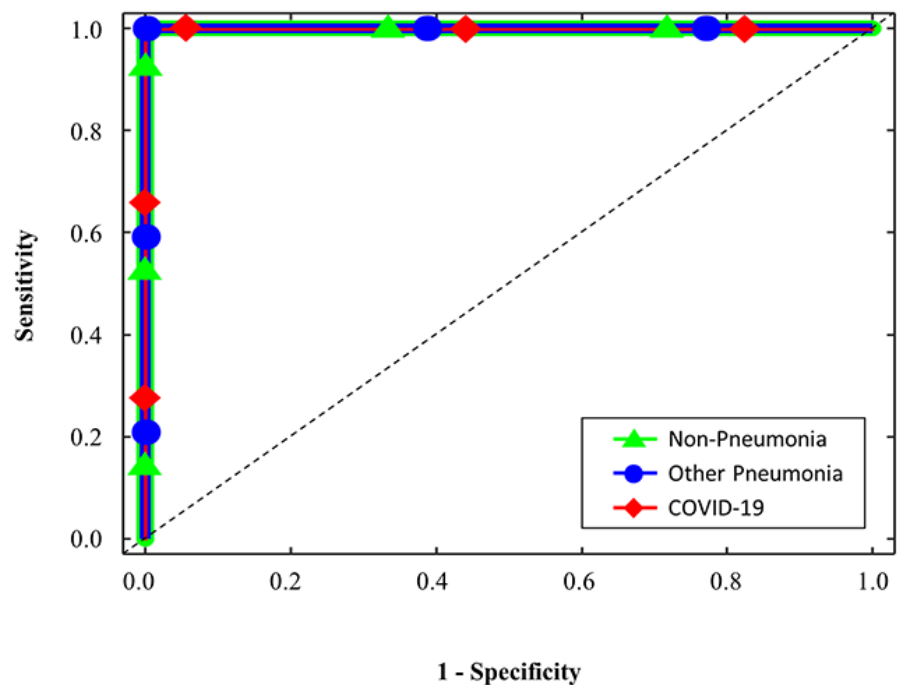


Figure 3. Confusion matrix and ROC curve in FCONet using VGG16; COVID-19: coronavirus disease; ROC: receiver operating characteristic.

Confusion Matrix (VGG16)

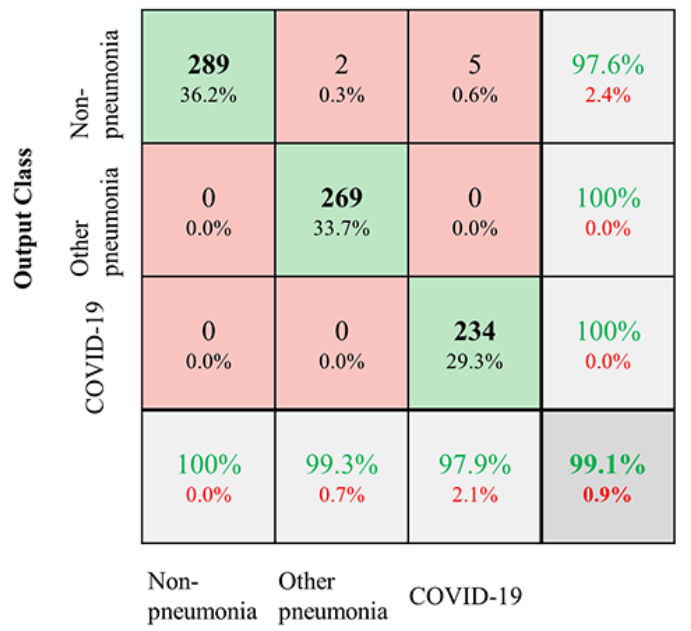

Target Class
ROC (VGG16)

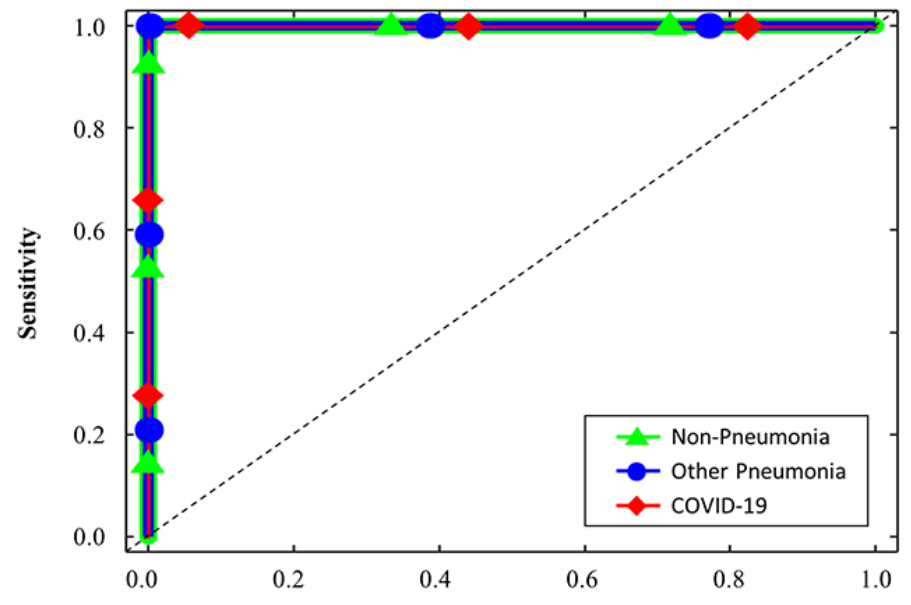

1 - Specificity

Figure 4. Confusion matrix and ROC curve in FCONet using Xception; COVID-19: coronavirus disease; ROC: receiver operating characteristic.

Confusion Matrix (Xception)

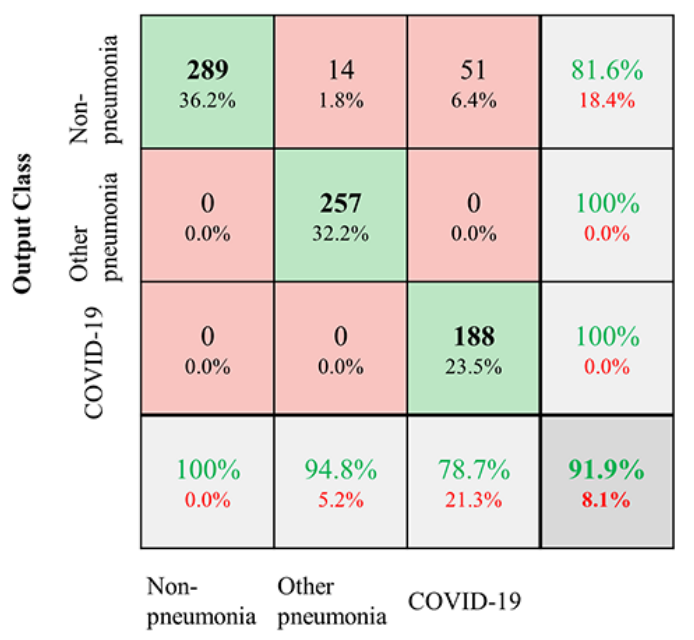

Target Class
ROC (Xception)

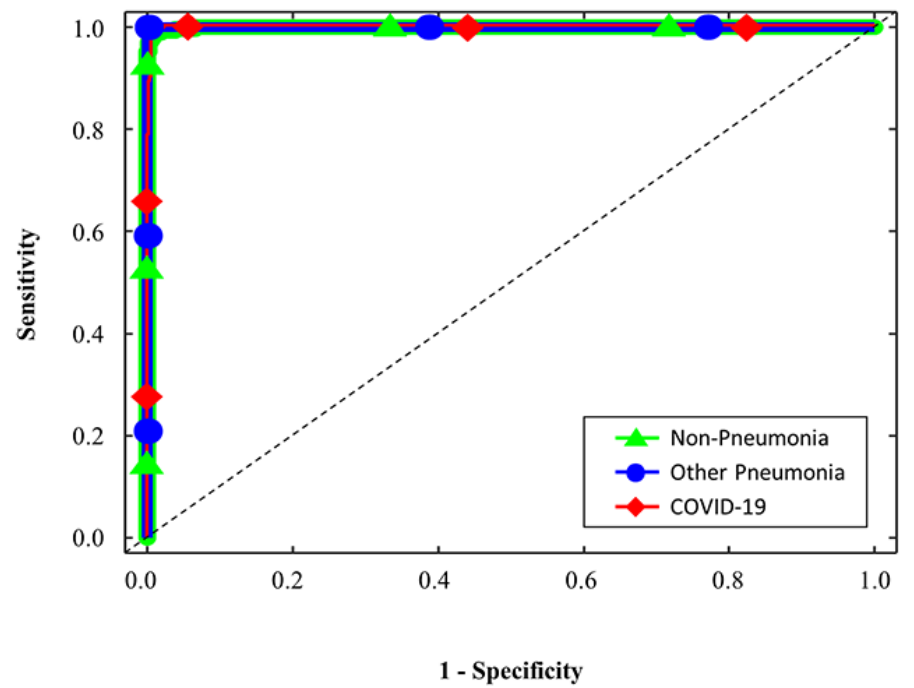

Figure 5. Confusion matrix and ROC curve in FCONet using Inception-v3; COVID-19: coronavirus disease; ROC: receiver operating characteristic. Confusion Matrix (InceptionV3)

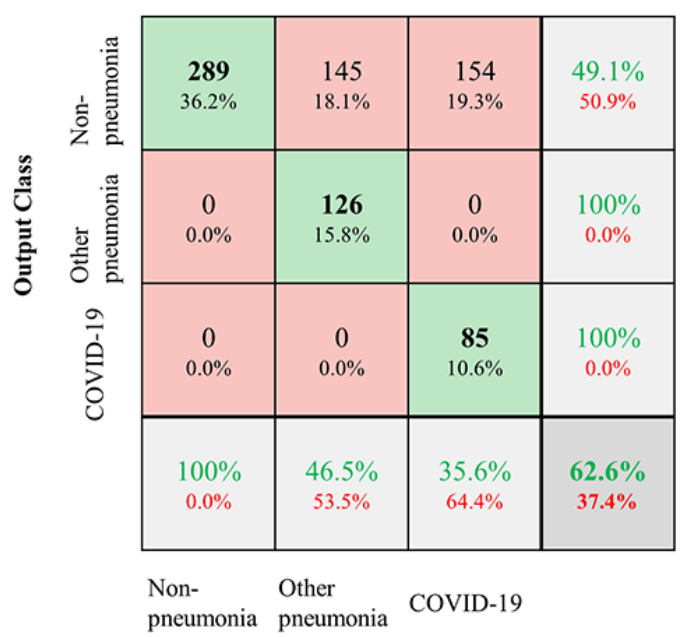

Target Class

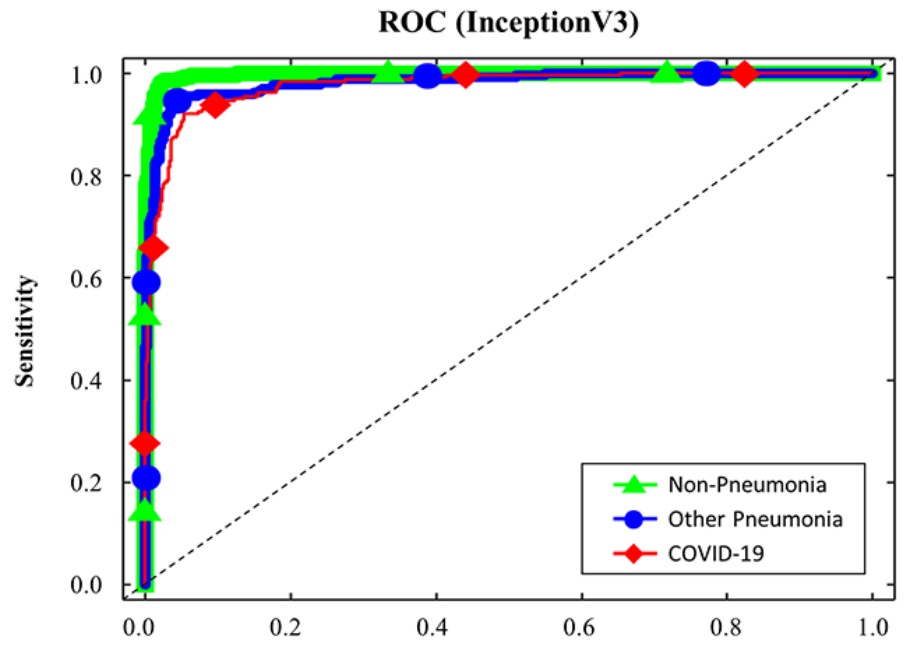

1 - Specificity 
Table 5. Performance of each deep learning model on the additional external validation data set of COVID-19 pneumonia images.

\begin{tabular}{ll}
\hline Model & Detection accuracy, \% \\
\hline ResNet-50 & 96.97 \\
VGG16 & 87.12 \\
Xception & 90.71 \\
Inception-v3 & 89.38 \\
\hline
\end{tabular}

To improve the interpretability of our model, we used the gradient-weighted class activation mapping (Grad-CAM) method [23] to visualize the important regions leading to the decision of FCONet. The model fully generates this localization map without the mapping annotation. The heatmaps (Figure 6) show the suspected regions for the examples of COVID-19, other pneumonia, and nonpneumonia. The heatmaps are standard jet colormaps and are overlapped on the original image, where red color highlights the activation region associated with the predicted class. More specifically, for the COVID-19 image group, the heatmap strongly indicated the suspected regions, as shown in examples from WKUH (Figure 6, top left), CNUH (Figure 6, top middle) and SIRM (Figure 6, top right). For the other pneumonia image groups, the heatmap demonstrated some suspected regions inside the lung area; the intensity was lower than that of the regions in the COVID-19 image group (Figure 6 , bottom left). For the healthy image group, there was no heatmap corresponding to the suspected regions (Figure 6, bottom middle). For the lung cancer images, the heatmap indicated some suspected regions inside the lung area; however, the intensity was also lower than that of the regions in the COVID-19 pneumonia group (Figure 6, bottom right).

Figure 6. Confusion matrice and ROC curve in FCONet using VGG16; COVID-19: coronavirus disease; ROC: receiver operating characteristic.
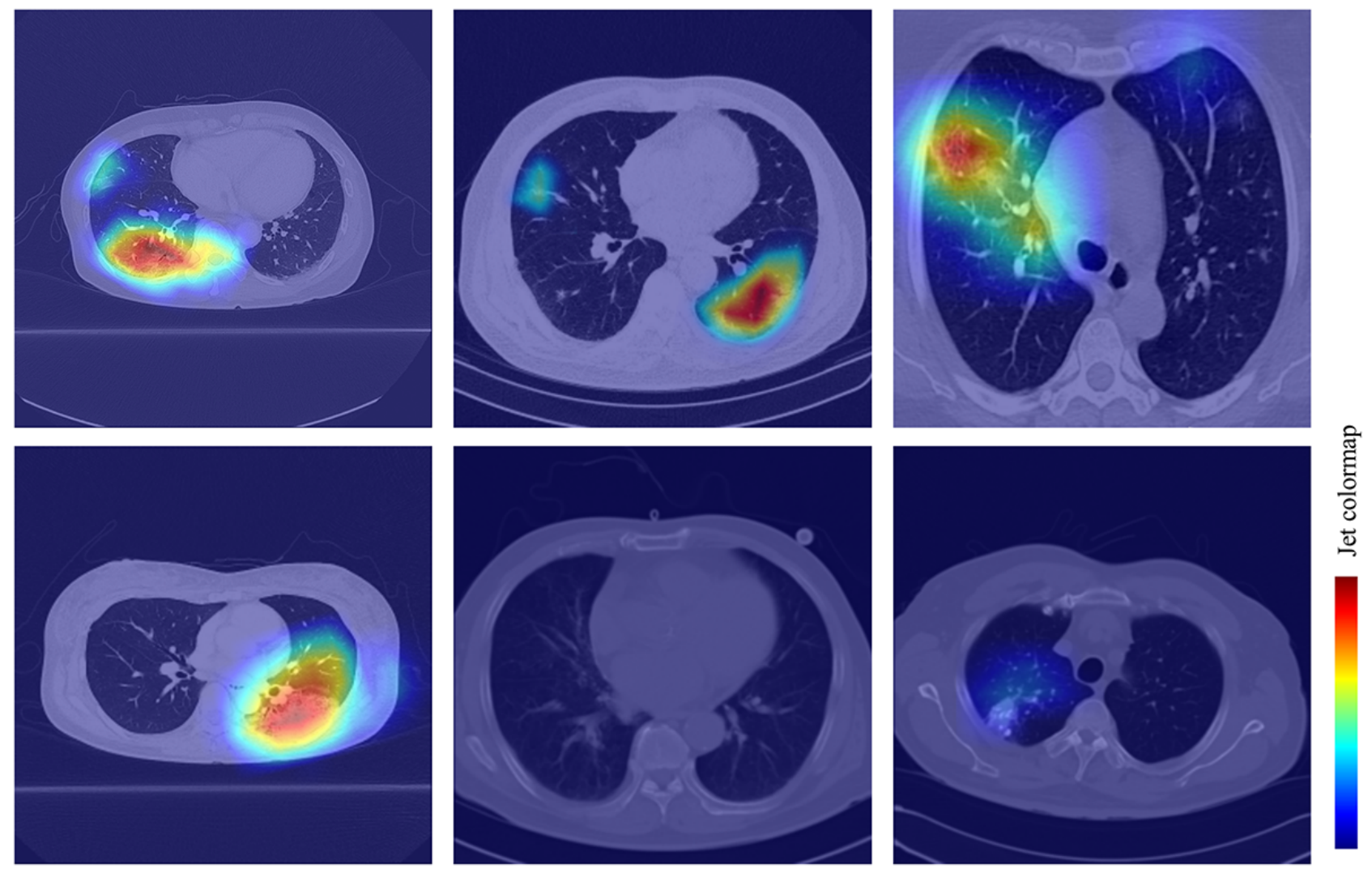

To test the generalizability of our proposed framework, we also trained and tested the models based on institutional data split for COVID-19 data: training data from CNUH and SIRM and tested data from WKUH. Because the COVID-19 data were split with a ratio of 65:35 (773 training data and 421 testing data for COVID-19), the other non-COVID-19 data were randomly split with the same ratio in a stratified fashion. Table 6 summarizes the performance of the FCONet framework. With ResNet-50, the sensitivity, specificity and accuracy for classifying COVID-19 pneumonia were $97.39 \%, 99.64 \%$ and
98.67\%, respectively $(P<.001)$. With VGG16, the sensitivity, specificity, and accuracy for classifying COVID-19 pneumonia were $97.15 \%, 99.64 \%$ and $98.57 \%$, respectively $(P<.001)$. With Xception, the sensitivity, specificity, and accuracy for classifying COVID-19 pneumonia were $90.50 \%, 94.82 \%$ and $92.97 \%$, respectively $(P<.001)$. With Inception-v3, the sensitivity, specificity, and accuracy for classifying COVID-19 pneumonia were $74.58 \%, 99.46 \%$ and $88.79 \%$, respectively $(P<.001)$. These results show that the FCONet framework can classify COVID-19 regardless of the data split approach. 
Table 6. Performance of the FCONet framework based on institutional data split for COVID-19 data.

\begin{tabular}{|c|c|c|c|c|c|}
\hline Model and data group & Sensitivity, $\%$ & Specificity, \% & Accuracy, $\%$ & $\mathrm{AUC}^{\mathrm{a}}$ & $P$ value \\
\hline ResNet-50 & & & & & $<.001$ \\
\hline COVID-19 pneumonia & 97.39 & 99.64 & 98.67 & 0.99 & \\
\hline Other pneumonia & 99.26 & 98.45 & 98.637 & 0.99 & \\
\hline Nonpneumonia & 100 & 100 & 100 & 1.0 & \\
\hline VGG16 & & & & & $<.001$ \\
\hline COVID-19 pneumonia & 97.15 & 99.64 & 98.57 & 0.99 & \\
\hline Other pneumonia & 99.26 & 98.31 & 98.57 & 0.99 & \\
\hline Nonpneumonia & 100 & 100 & 100 & 1.0 & \\
\hline Xception & & & & & $<.001$ \\
\hline COVID-19 pneumonia & 90.50 & 94.82 & 92.97 & 0.98 & \\
\hline Other pneumonia & 89.30 & 94.37 & 92.97 & 0.98 & \\
\hline Nonpneumonia & 100 & 100 & 100 & 1.0 & \\
\hline Inception-v3 & & & & & $<.001$ \\
\hline COVID-19 pneumonia & 74.58 & 99.46 & 88.79 & 0.98 & \\
\hline Other pneumonia & 97.42 & 84.93 & 88.38 & 0.97 & \\
\hline Nonpneumonia & 100 & 99.42 & 99.59 & 0.99 & \\
\hline
\end{tabular}

${ }^{\mathrm{a}} \mathrm{AUC}$ : area under the curve.

\section{Discussion}

\section{Principal Findings}

We were able to develop the FCONet deep learning models to diagnose COVID-19 pneumonia in a few weeks using transfer learning based on pretrained models. The FCONet based on ResNet-50 showed excellent diagnostic performance to detect COVID-19 pneumonia. Although the diagnostic accuracy of the FCONet models based on VGG16, ResNet-50, and Xception was excellent in the testing data set (sensitivity, 97.91\%, 100\%, and $97.91 \%$, respectively; specificity, $100 \%, 99.64 \%$ and $99.29 \%$, respectively), external validation using the low-quality image data set demonstrated that detection accuracy was the highest with ResNet-50 (96.97\%), followed by Xception (90.71\%), Inception-v3 (89.38\%), and VGG16 (87.12\%).

To collect as many images as possible within a limited time, we collected readily available chest CT images of COVID-19 patients from institutions in our region (WKUH and CNUH) and a public COVID-19 database established by SIRM. We also systematically searched for chest CT images of COVID-19 embedded in recent papers published between January 19 and March 25, 2020. As these CT images in the published paper were of low quality, we used them only in an additional external validation data set.

During a national crisis such as the COVID-19 pandemic, when the number of infected patients is precipitously increasing and physicians are occupied combating the disease, rapid development of AI methods to detect COVID-19 in CT is crucial to alleviate the clinical burden of physicians and to increase the efficiency of the patient management process [8]. However, significant challenges remain when developing such AI techniques within a limited time to collect CT data and train AI models.

To save time for AI training, we used the chest CT images directly without preprocessing of the lung segmentation. In general, lung segmentation preprocessing is regarded to improve the accuracy of AI training [24-27]; we believe that this improvement can be traded off in exchange for saving time. For AI training, we chose the transfer learning algorithms. Transfer learning enabled us to save time by using pretrained CNN models in the ImageNet data sets, including VGG16, ResNet-50, Inception-v3, and Xception [28]. In our study, FCONet based on ResNet-50 showed excellent results and outperformed the FCONet models based on the other three pretrained models in both our testing data set and the additional external validation data set. The VGG model is regarded as a traditional sequential network architecture and may be hampered by slow training and a large model size [17]. The ResNet-50 model is characterized by network-in-network architectures, which have much deeper layers than those of VGG models, enabling reduction of the model size [18]. Our results suggest that transfer learning for a 2D deep learning framework can be robustly applied to deep learning models and that the ResNet-50 model provides the best accuracy.

We adopted AI training based on a 2D image framework rather than a 3D framework because 3D deep learning requires significantly higher computation power than sequential 2D image analyses [29]. In our emergent clinical setting to fight COVID-19, a simple and rapid model may be preferable to a complex and slow model. In addition, training a 2D image framework saves time for AI development. 
Despite limited resources and time, we were able to generate a deep learning model to detect COVID-19 from chest CT with excellent diagnostic accuracy. To date, a few papers have been published on AI models for detecting COVID-19 in chest CT images [6]. An AI model named COVNet was trained using 4356 CT images from six hospitals in China. It showed 90\% sensitivity (95\% CI 83\%-94\%) and 96\% specificity (95\% CI 93\%-98\%) in detecting COVID-19, which is comparable with our results. However, we cannot compare our FCONet to COVNet because the training and testing data sets are different.

Although chest radiography is the most commonly used imaging tool to detect COVID-19, its sensitivity is lower than that of CT [30]. However, in this pandemic period, clinicians may hesitate to perform chest $\mathrm{CT}$ due to limited resources such as CT scanners and radiologists as well as contamination of CT scanners [31]. In our hospitals (WKUH and CNUH), we recently dedicated a mobile CT scanner exclusively to COVID-19 patients to alleviate the physical and mental stress of medical staff. We believe that incorporating an AI model to detect suspicious lesions of COVID-19 pneumonia can improve the workflow by providing rapid diagnostic support.

\section{Limitations and Future Work}

Our study has several limitations. Firstly, our AI models were validated mainly using a split testing data set. Thus, the testing data set was obtained from the same sources as the training data set. This may raise issues of generalizability and overfitting of our models [32,33]. Indeed, the detection accuracy of our model decreased slightly for the external validation data set using chest CT images from published papers. However, the initial goal was to incorporate a deep learning model in our emergent clinical setting as a supporting tool. In the near future, we will train our model using CT images from various institutions and countries. Secondly, we used a relatively small amount of data to train the deep learning models. Thus, we will establish a sustainable AI training system that can continue to train our model using prospectively collected CT images.

\section{Conclusions}

We described FCONet, a simple 2D deep learning framework based on a single chest CT image, as a diagnostic aid that provides excellent diagnostic performance to diagnose COVID-19 pneumonia. The FCONet model based on ResNet-50 appears to be the best model, outperforming other models based on VGG16, Xception, and Inception-v3.

\section{Acknowledgments}

This work was partially supported by the National Research Foundation of Korea, Ministry of Science, ICT, and Future Planning, through the Basic Science Research Program (grant number: NRF-2020R1A2C1014829), and from the Korea Health Industry Development Institute (grant number: HI18C2383 and HI18C1216).

\section{Conflicts of Interest}

None declared.

\section{References}

1. Coronavirus disease 2019 (COVID-19) Situation Report - 84. World Health Organization. 2020 Apr 13. URL: https://www. who.int/docs/default-source/coronaviruse/situation-reports/20200413-sitrep-84-covid-19.pdf?sfvrsn=44f511ab 2 [accessed 2020-06-23]

2. Bhatraju PK, Ghassemieh BJ, Nichols M, Kim R, Jerome KR, Nalla AK, et al. Covid-19 in Critically Ill Patients in the Seattle Region - Case Series. N Engl J Med 2020 May 21;382(21):2012-2022 [FREE Full text] [doi: 10.1056/NEJMoa2004500] [Medline: 32227758]

3. Loeffelholz MJ, Tang Y. Laboratory diagnosis of emerging human coronavirus infections - the state of the art. Emerg Microbes Infect 2020 Dec;9(1):747-756 [FREE Full text] [doi: 10.1080/22221751.2020.1745095] [Medline: 32196430]

4. Li Y, Yao L, Li J, Chen L, Song Y, Cai Z, et al. Stability issues of RT-PCR testing of SARS-CoV-2 for hospitalized patients clinically diagnosed with COVID-19. J Med Virol 2020 Jul;92(7):903-908 [FREE Full text] [doi: 10.1002/jmv.25786] [Medline: 32219885]

5. Ai T, Yang Z, Hou H, Zhan C, Chen C, Lv W, et al. Correlation of Chest CT and RT-PCR Testing in Coronavirus Disease 2019 (COVID-19) in China: A Report of 1014 Cases. Radiology 2020 Feb 26:200642 [FREE Full text] [doi: 10.1148/radiol.2020200642] [Medline: 32101510]

6. Rubin GD, Ryerson CJ, Haramati LB, Sverzellati N, Kanne JP, Raoof S, et al. The Role of Chest Imaging in Patient Management during the COVID-19 Pandemic: A Multinational Consensus Statement from the Fleischner Society. Radiology $2020 \mathrm{Jul}$;296(1):172-180 [FREE Full text] [doi: 10.1148/radiol.2020201365] [Medline: 32255413]

7. Vashist SK. In Vitro Diagnostic Assays for COVID-19: Recent Advances and Emerging Trends. Diagnostics (Basel) 2020 Apr 05;10(4):202 [FREE Full text] [doi: 10.3390/diagnostics10040202] [Medline: 32260471]

8. Li D, Wang D, Dong J, Wang N, Huang H, Xu H, et al. False-Negative Results of Real-Time Reverse-Transcriptase Polymerase Chain Reaction for Severe Acute Respiratory Syndrome Coronavirus 2: Role of Deep-Learning-Based CT Diagnosis and Insights from Two Cases. Korean J Radiol 2020 Apr;21(4):505-508 [FREE Full text] [doi: $\underline{10.3348 / \mathrm{kjr} .2020 .0146]}$ [Medline: $\underline{32174053}$ ] 
9. Long C, Xu H, Shen Q, Zhang X, Fan B, Wang C, et al. Diagnosis of the Coronavirus disease (COVID-19): rRT-PCR or CT? Eur J Radiol 2020 May;126:108961 [FREE Full text] [doi: 10.1016/j.ejrad.2020.108961] [Medline: 32229322]

10. Mossa-Basha M, Medverd J, Linnau K, Lynch JB, Wener MH, Kicska G, et al. Policies and Guidelines for COVID-19 Preparedness: Experiences from the University of Washington. Radiology 2020 Apr 08:201326 [FREE Full text] [doi: 10.1148/radiol.2020201326] [Medline: 32267209]

11. Bai HX, Hsieh B, Xiong Z, Halsey K, Choi JW, Tran TML, et al. Performance of radiologists in differentiating COVID-19 from viral pneumonia on chest CT. Radiology 2020 Mar 10:200823 [FREE Full text] [doi: 10.1148/radiol.2020200823] [Medline: 32155105]

12. Li L, Qin L, Xu Z, Yin Y, Wang X, Kong B, et al. Artificial Intelligence Distinguishes COVID-19 from Community Acquired Pneumonia on Chest CT. Radiology 2020 Mar 19:200905 [FREE Full text] [doi: 10.1148/radiol.2020200905] [Medline: 32191588]

13. COVID-19 Database. Italian Society of Medical and Interventional Radiology (SIRM). URL: https://www.sirm.org/en/ category/articles/covid-19-database/ [accessed 2020-06-23]

14. Yang X, He X, Zhao J, Zhang Y, Zhang S, Xie P. COVID-CT-dataset: a CT scan dataset about COVID-19. arXiv. 2020 Mar 20. URL: https://arxiv.org/abs/2003.13865 [accessed 2020-06-23]

15. Wormanns D, Fiebich M, Saidi M, Diederich S, Heindel W. Automatic detection of pulmonary nodules at spiral CT: clinical application of a computer-aided diagnosis system. Eur Radiol 2002 May;12(5):1052-1057. [doi: 10.1007/s003300101126] [Medline: 11976846$]$

16. Ohana M, Labani A, Severac F, Jeung M, Gaertner S, Caspar T, et al. Single source dual energy CT: What is the optimal monochromatic energy level for the analysis of the lung parenchyma? Eur J Radiol 2017 Mar;88:163-170. [doi: 10.1016/j.ejrad.2017.01.016] [Medline: 28189203]

17. Simonyan K, Zisserman A. Very deep convolutional networks for large-scale image recognition. arXiv. 2014. URL: https:/ larxiv.org/abs/1409.1556 [accessed 2020-06-23]

18. He K, Zhang X, Ren S, Sun J. Deep Residual Learning for Image Recognition. 2016 Presented at: 2016 IEEE Conference on Computer Vision and Pattern Recognition (CVPR); June 26-July 1, 2016; Las Vegas, NV. [doi: 10.1109/CVPR.2016.90]

19. Szegedy C, Vanhoucke V, Ioffe S, Shlens J, Wojna Z. Rethinking the Inception Architecture for Computer Vision. 2016 Presented at: 2016 IEEE Conference on Computer Vision and Pattern Recognition (CVPR); June 26-July 1, 2016; Las Vegas, NV. [doi: 10.1109/cvpr.2016.308]

20. Chollet F. Xception: Deep Learning with Depthwise Separable Convolutions. 2017 Presented at: 017 IEEE Conference on Computer Vision and Pattern Recognition (CVPR); July 21-26, 2017; Honolulu, HI. [doi: 10.1109/cvpr.2017.195]

21. Rawat W, Wang Z. Deep Convolutional Neural Networks for Image Classification: A Comprehensive Review. Neural Comput 2017 Sep;29(9):2352-2449. [doi: 10.1162/neco a 00990]

22. Kingma DP, Ba JL. Adam: A method for stochastic optimization. 2015 Presented at: 3rd International Conference for Learning Representations; May 7-9, 2015; San Diego, CA URL: https://arxiv.org/pdf/1412.6980.pdf

23. Selvaraju R, Cogswell M, Das A, Vedantam R, Parikh D, Batra D. Grad-CAM: Visual Explanations from Deep Networks via Gradient-Based Localization. : Visual explanations from deep networks via gradient-based localization. Proceedings of the IEEE international conference on computer vision; 2017 Presented at: 2017 IEEE International Conference on Computer Vision (ICCV); Oct. 22-29, 2017; Venice, Italy. [doi: 10.1109/iccv.2017.74]

24. Li L, Qin L, Xu Z, Yin Y, Wang X, Kong B, et al. Artificial Intelligence Distinguishes COVID-19 from Community Acquired Pneumonia on Chest CT. Radiology 2020 Mar 19:200905 [FREE Full text] [doi: 10.1148/radiol.2020200905] [Medline: $\underline{\text { 32191588] }}$

25. Xu X, Jiang X, Ma C, Du P, Li X, Lv S, et al. Deep learning system to screen coronavirus disease 2019 pneumonia. arXiv. URL: https://arxiv.org/abs/2002.09334 [accessed 2020-06-23]

26. Zheng C, Deng X, Fu Q, Zhou Q, Feng J, Ma H, et al. Deep Learning-based Detection for COVID-19 from Chest CT using Weak Label. medRxiv 2020 Mar 26:preprint. [doi: 10.1101/2020.03.12.20027185]

27. Chung H, Ko H, Jeon SJ, Yoon K, Lee J. Automatic Lung Segmentation With Juxta-Pleural Nodule Identification Using Active Contour Model and Bayesian Approach. IEEE J Transl Eng Health Med 2018;6:1-13. [doi: 10.1109/jtehm.2018.2837901]

28. Deng J, Dong W, Socher R, Li L, Li K, Fei-Fei L. ImageNet: A large-scale hierarchical image database. Imagenet: A large-scale hierarchical image database; 2009 Presented at: 2009 IEEE Conference on Computer Vision and Pattern Recognition; June 20-25, 2009; Miami, FL p. A. [doi: 10.1109/cvpr.2009.5206848]

29. Maturana D, Scherer S. VoxNet: A 3D Convolutional Neural Network for real-time object recognition. 2015 Presented at: 2015 IEEE/RSJ International Conference on Intelligent Robots and Systems (IROS); 28 September-3 October 2015; Hamburg, Germany. [doi: 10.1109/iros.2015.7353481]

30. Yoon SH, Lee KH, Kim JY, Lee YK, Ko H, Kim KH, et al. Chest Radiographic and CT Findings of the 2019 Novel Coronavirus Disease (COVID-19): Analysis of Nine Patients Treated in Korea. Korean J Radiol 2020 Apr;21(4):494-500 [FREE Full text] [doi: 10.3348/kjr.2020.0132] [Medline: 32100485] 
31. Mossa-Basha M, Meltzer CC, Kim DC, Tuite MJ, Kolli KP, Tan BS. Radiology Department Preparedness for COVID-19: Scientific Expert Panel. Radiology 2020 Mar 16:200988 [FREE Full text] [doi: 10.1148/radiol.2020200988] [Medline: 32175814]

32. Kim DW, Jang HY, Kim KW, Shin Y, Park SH. Design Characteristics of Studies Reporting the Performance of Artificial Intelligence Algorithms for Diagnostic Analysis of Medical Images: Results from Recently Published Papers. Korean J Radiol 2019 Mar;20(3):405-410 [FREE Full text] [doi: 10.3348/kjr.2019.0025] [Medline: 30799571]

33. Santosh KC. AI-Driven Tools for Coronavirus Outbreak: Need of Active Learning and Cross-Population Train/Test Models on Multitudinal/Multimodal Data. J Med Syst 2020 Mar 18;44(5):93 [FREE Full text] [doi: 10.1007/s10916-020-01562-1] [Medline: $\underline{32189081]}$

\author{
Abbreviations \\ AI: artificial intelligence \\ ANOVA: analysis of variance \\ API: application programming interface \\ AUC: area under the curve \\ CNN: convolutional neural networks \\ CNUH: Chonnam National University Hospital \\ COVID-19: coronavirus disease \\ CT: computed tomography \\ DICOM: Digital Imaging and Communications in Medicine \\ FCONet: fast-track COVID-19 classification network \\ FN: false negative \\ FP: false positive \\ Grad-CAM: gradient-weighted class activation mapping \\ ROC: receiver operating characteristic \\ SARS-CoV-2: severe acute respiratory syndrome coronavirus 2 \\ SIRM: Italian Society of Medical and Interventional Radiology \\ TN: true negative \\ TP: true positive \\ WKUH: Wonkwang University Hospital
}

\author{
Edited by G Eysenbach; submitted 23.04.20; peer-reviewed by J Lee, T Goodwin; comments to author 14.05.20; revised version \\ received 31.05.20; accepted 21.06.20; published 29.06.20 \\ Please cite as: \\ Ko H, Chung H, Kang WS, Kim KW, Shin Y, Kang SJ, Lee JH, Kim YJ, Kim NY, Jung H, Lee J \\ COVID-19 Pneumonia Diagnosis Using a Simple 2D Deep Learning Framework With a Single Chest CT Image: Model Development \\ and Validation \\ J Med Internet Res 2020;22(6):e19569 \\ URL: http://www.jmir.org/2020/6/e19569/ \\ doi: $10.2196 / 19569$ \\ PMID: $\underline{32568730}$
}

(CHoon Ko, Heewon Chung, Wu Seong Kang, Kyung Won Kim, Youngbin Shin, Seung Ji Kang, Jae Hoon Lee, Young Jun Kim, Nan Yeol Kim, Hyunseok Jung, Jinseok Lee. Originally published in the Journal of Medical Internet Research (http://www.jmir.org), 29.06.2020. This is an open-access article distributed under the terms of the Creative Commons Attribution License (https://creativecommons.org/licenses/by/4.0/), which permits unrestricted use, distribution, and reproduction in any medium, provided the original work, first published in the Journal of Medical Internet Research, is properly cited. The complete bibliographic information, a link to the original publication on http://www.jmir.org/, as well as this copyright and license information must be included. 